\title{
Navigation : Préhistoire
}

\author{
Jorge Onrubia-Pintado
}

\section{OpenEdition}

Journals

Édition électronique

URL : https://journals.openedition.org/encyclopedieberbere/2686

DOI : 10.4000/encyclopedieberbere.2686

ISSN : 2262-7197

\section{Éditeur}

Peeters Publishers

\section{Édition imprimée}

Date de publication : 4 octobre 2012

Pagination : 5295-5299

ISBN : 978-90-429-2640-0

ISSN : 1015-7344

\section{Référence électronique}

Jorge Onrubia-Pintado, « Navigation : Préhistoire », Encyclopédie berbère [En ligne], 33 | 2012, document N19, mis en ligne le 23 novembre 2020, consulté le 17 février 2022. URL : http:// journals.openedition.org/encyclopedieberbere/2686; DOI : https://doi.org/10.4000/ encyclopedieberbere. 2686

Ce document a été généré automatiquement le 17 février 2022.

(c) Tous droits réservés 


\title{
Navigation : Préhistoire
}

\author{
Jorge Onrubia-Pintado
}

1 Quels que soient la région et le milieu aquatique (continental ou marin) concernés, l'examen de la question des navigations préhistoriques pose un certain nombre de problèmes d'ordre méthodologique dont il faut tenir compte. D'une part, les difficultés traditionnellement liées à la visibilité des vestiges archéologiques, qui pour cette période constituent les seules preuves susceptibles d'attester une éventuelle relation entre les groupes humains et les eaux marines ou intérieures, sont accentuées dans le cas des mers et des océans par les profondes modifications qu'ils ont subies au cours de la Pré-histoire. La plupart de ces altérations, dont on peut citer en guise d'exemple la baisse d'une centaine de mètres du niveau marin lors du maximum de la dernière période glaciaire (20.000 ans B.P.), sont le résultat de changements climatiques et océanographiques globaux qui, en outre, ne sont pas sans conséquences sur les conditions de navigation alors existantes : vents, courants, émergence de fonds marins aujourd'hui noyés...

2 Pour ce qui est du nord de l'Afrique, les témoignages archéologiques de l'existence de navigations préhistoriques en milieu fluvial ou lacustre sont limités. En effet, à l'heure actuelle les seules preuves seraient fournies par les embarcations néolithiques en tiges végétales figurées sur les parois rocheuses du Tassili des Ajjer, dont la technique est encore vivante au lac Tchad et existait, jusqu'au début du siècle dernier, dans la région marocaine de l'oued Loukkous (Guerrero Ayuso 2009, p. 179-180). Il est évident que, même si les donnés archéologiques font cruellement défaut pour la période concernée, cette tradition de navigations dans les eaux continentales devait être jadis assez répandue. Par exemple, d'après Léon l'Africain (1980, t. II, p. 543), de grands radeaux pourvus d'outres gonflées étaient employés au XVIe siècle par les habitants de la région d'Azemmour pour traverser les flots de l'oued Oum Errabia lors des périodes de crues.

Ceci dit, l'essentiel des arguments susceptibles de prouver l'existence d'une quelconque activité nautique dans la Préhistoire nord-africaine provient du milieu marin. Mais nous ne disposons d'aucune preuve directe: restes conservés d'embarcations ou représentations iconographiques sûres et bien datées. Il faut donc se contenter de témoignages indirects. Faute d'une étude d'ensemble sur la pêche préhistorique qui, à 
partir de déterminations ichtyo-archéologiques fiables, permettrait de déduire l'usage incontestable de moyens de navigation, ces preuves sont uniquement étayées par les évidences archéologiques du franchissement des bras de mer séparant l'Afrique et l'Europe et par la fréquentation et colonisation de certaines îles.

4 La question de la traversée éventuelle du détroit de Gibraltar et du canal siculo-tunisien au cours du Paléolithique ancien est un vieux débat qui est loin d'être définitivement clos. Ces derniers temps, la datation de 1,8 million d'années du site d'Aïn Hanech (Algérie), la certitude d'une présence humaine dans les péninsules Ibérique et Italique il y a plus d'un million d'années et, enfin, les arguments fournis par la colonisation de lî̂le de Flores (Indonésie), atteinte vers 800.000 ans avant nos jours après une navigation d'une vingtaine de kilomètres, ont contribué à donner un nouveau élan à l'hypothèse d'un peuplement de l'Europe par des Homo ergaster africains (jadis appelés Homo erectus, terme aujourd'hui réservé aux seuls fossiles asiatiques) ayant traversé la Méditerranée. Si séduisante que cette possibilité puisse paraître, toutes les preuves archéologiques aujourd'hui disponibles semblent l'écarter au profit de la voie de peuplement terrestre à travers le Proche Orient et l'Europe orientale (Broodbank 2006).

5 La situation n'est pas moins polémique en ce qui concerne les éventuelles relations euro-africaines pendant le Paléolithique moyen et supérieur. L'idée d'un franchissement du détroit de Gibraltar par les Atériens du nord de l'actuel Maroc (Kozłowski et al. 2004), chassés par l'aridité qui s'est installée dans la région à la fin du dernier maximum glaciaire, n'est qu'une hypothèse qui bénéficie, certes, de la parenté morphologique entre leurs industries lithiques et celles du Solutréen ibérique et de sa coïncidence avec un moment de bas niveau marin (-100/-120 m par rapport à factuel). Celui-ci a, en effet, profondément modifié la paléogéographie littorale de la région, rapprochant les deux côtes et faisant émerger quelques hauts fonds marins susceptibles d'en faciliter le passage.

6 Comme d'aucuns le pensent, il est possible que cette altération glacio-eustatique, qui a provoqué l'émergence d'importantes extensions du plateau continental actuellement noyées, soit aussi à l'origine de l'absence quasi absolue de traces de contacts entre le nord de l'Afrique et le sud de l'Europe pendant l'Ibéromaurusien. Mais, même si le harpon magdalénien (14.000 ans B.P.) trouvé dans la grotte marocaine de Taforalt pouvait constituer une preuve incontestable de ces relations, tout porte à penser que les sociétés méditerranéennes du Paléolithique supérieur étaient plutôt "terriennes » (Broodbank 2006). D'ailleurs la thèse d'une genèse italique des mechtoïdes ibéromaurusiens proposée par D. Ferembach, relayée par la suite par A. Debénath (cf. Onrubia Pintado 1992), n'a jamais dépassé le stade d'une suggestion quelque peu provocante.

7 Le panorama n'est certes plus le même à l'Epipaléolithique (Capsien et Épipaléolithique méditerranéen) et au Néolithique, moment où se produit une vraie éclosion du phénomène maritime en Méditerranée (Broodbank 2006). Dans le Maghreb oriental, l'obsidienne* provenant de Pantelleria et peut-être aussi de Lipari, trouvée dans des sites épipaléolithiques qui font l'objet d'une néolithisation progressive, atteste la fréquentation de ce littoral par des navigateurs dès la moitié du VII ${ }^{e}$ millénaire avant l'ère chrétienne (Mulazzani et al. 2010a et 2010b). Quelques siècles plus tard, à l'autre extrémité du Maghreb, la néolithisation de la région littorale comprise entre la péninsule Tingitane et l'Oranais est sans doute aussi le résultat d'une dynamique de 
colonisation maritime qui embrasse, au même moment, l'Andalousie et le sud du Portugal (Linstädter 2008).

8 À partir de ce moment, les évidences de contacts euro-africains, et donc de navigations, se multiplient jusqu'au moment où l'on assiste aux premières activités des marins et des flottes de l'Antiquité. Dans la région du détroit de Gibraltar, les relations néolithiques entre les deux rives se prolongent au Chalcolithique et pendant tout l'Âge du Bronze, sans doute, au moins en partie, sur la base de l'échange de matières premières telles que l'ivoire et la coquille d'œuf* d'autruche* (Onrubia Pintado 1988). Au Maghreb oriental, les témoignages de ces navigations post-néolithiques sont plus rares, mais il est fort possible que l'origine des haouanet (petits hypogées) doive être mise en rapport avec l'existence, pendant la fin de l'Âge du Bronze, de liaisons maritimes entre cette région, la Sardaigne et la Sicile (Camps 2000). De façon significative, deux de ces chambres funéraires creusées dans le roc sont décorées de figurations de bateaux dont l'attribution chronologique s'étalerait, suivant les avis divergents des spécialistes, entre un horizon nettement pré-phénicien et une phase franchement libyco-punique (Longerstay 2000, Guerrero Ayuso 2008, Medas 2008).

En toute logique, de l'éclosion néolithique de la vie et des navigations maritimes date aussi la fréquentation et le peuplement de certains îles et îlots situés au large des côtes nord-africaines. Pour ce qui est de la Méditerranée, c'est le cas, par exemple, des îles Chafarinas (transcription espagnole du mot rifain ichfaren, "les littoraux »), occupées dès la moitié du $\mathrm{V}^{\mathrm{e}}$ millénaire avant l'ère chrétienne (Rojo et al. 2006) et, en ce qui concerne l'Atlantique, de Mogador, de Herné ou, enfin, des îles et des îlots de la côte mauritanienne comme Tidra ou le banc d'Arguin (cf. Onrubia-Pintado 1997; Vernet 2007). Mais il est fort possible que certaines de ces îles étaient rattachées au continent à ce moment, et donc accessibles à pied sec. Quant aux autres, leur disposition assez abritée, leur proximité par rapport à la ligne de rivage de l'époque, comparable à l'actuelle, et les hauts fonds marins qui les entourent ne semblent pas demander pour les atteindre le concours de techniques nautiques sophistiquées.

Un cas bien différent est sans doute celui que représentent les îles Canaries, dont le peuplement ne fut possible que grâce à une navigation au grand cabotage. C'est pour cela que, même si une première fréquentation par les pêcheurs et les ramasseurs de coquillages néolithiques du littoral saharien voisin ne peut pas être définitivement écartée (Onrubia-Pintado 1997), tous les indices aujourd'hui disponibles concordent pour accorder le rôle majeur de cette exploration et colonisation à des populations disposant d'une technologie nautique avancée. De toute évidence, celle-ci ne saurait être indépendante des progrès apportés par les marines de l'Antiquité (Guerrero Ayuso 2008, Medas 2008, Tejera et Chávez 2011).

\section{BIBLIOGRAPHIE}

BROODBANK C., 2006 - « The Origins and Early Development of Mediterranean Maritime Activity », Journal of Mediterranean Archaeology, 19-2, p. 199-230. 
CAMPS G., 2000 - « H25. Haouanet. Petits hypogées de l'Afrique du Nord », in Encyclopédie berbère, XXII (Hadrumetum-Hidjaba), Aix-en-Provence, Édisud, p. 3361-3374.

GUERRERO AYUSO V. M, 2008 - « "Las Naves de Kerné” (II). Navegando por el Atlántico durante la protohistoria y la antigüedad », in R. González Antón, F. López Pardo et V. Peña Romo (éds.), Los fenicios y el Atlántico (IV Coloquio del CEFYP), Madrid, Centro de Estudios Fenicios y Púnicos, p. 69-142.

GUERRERO AYUSO V. M., 2009 - Prehistoria de la navegación. Origen y desarrollo de la arquitectura naval primigenia (BAR International Series 1952), Oxford, Archaeopress.

KOzłowsKi J., BOUZzoUGGAR A. et OTTE M., 2004 - «Étude des ensembles lithiques atériens de la grotte d'El Aliya à Tanger (Maroc) », in M. Otte, A. Bouzzouggar et J. KOZŁowSKI (dir.), La préhistoire de Tanger (ERAUL, 105), Liège, Université de Liège, p. 42-89.

LÉON L'AFRICAIN J., 1981 - Description de l'Afrique (Publ. de l'Institut des Hautes Études Marocaines, LXI), Paris, Maisonneuve. 2 vol. (nouv. éd. traduite de l'italien par A. Épaulard et annotée par A. Épaulard, Th. Monod, H. Lhote et R. Mauny).

LINSTÄDTER J., 2008 - « The Epipalaeolithic-Neolithic-Transition in the Mediterranean region of Northwest Africa », Quartär, 55, p. 41-62.

LONGERSTAY M., 2000 - «H25. Haouanet. La décoration des haouanet », in Encyclopédie berbère, XXII (Hadrumetum-Hidjaba), Aix-en-Provence, Édisud, p. 3374-3386.

MEDAS S., 2008 - « La navigazione antica lungo le coste atlantiche dell'Africa e verso le Isole Canarie. Analisi della componente nautica a confronto con le esperienze medievali », in R. González Antón, F. López Pardo et V. Peña Romo (éd.), Los fenicios y el Atlántico (IV Coloquio del CEFYP), Madrid, Centro de Estudios Fenicios y Púnicos, p. 143-215.

MULAZZANI S., CAVULLI F., AOUADI-ABDELJAOUAD N., BELHOUCHET L., BOUSSOFFARA R., CURCI A., DRIDI Y., JEDDI Z., LE BOURDONNEC F.-X., MAINI E., MAGNANI G., MANNINO M. A., POUPEAU G. et TOSI M, 2010a - « Il sito olocenico costiero SHM-1 (Hergla, Tunisia), tra sostrato capsiano e le prime attestazioni della più antica navigazione ", Rivista di Scienze Preistoriche, LX, p. 49-61.

MULAZZANI S., LE BOURDONNEC F.-X., BELHOUCHET L., POUPEAU G., ZOUGHLAMI J., DUBERNET S., TUFANO E., LEFRAIS Y. et KHEDHAIER R, 2010b - «Obsidian from the Epipalaeolithic and Neolithic eastern Maghreb. A view from the Hergla context (Tunisia) ", Journal of Archaeological Science, 37, p. 2529-2537.

ONRUBIA-PINTADO J., 1988 - « Modalidades, implicaciones y significación de las relaciones prehistóricas ibero-magrebíes. Problemas y perspectivas », in E. Ripoll Perelló (éd.), Actas del Congreso Internacional "El Estrecho de Gibraltar" (Ceuta, novembre 1987), Madrid, UNEDAyuntamiento de Ceuta, t. I, p. 147-171.

ONRUBLA-PINTADO J., 1992 - « Las primeras navegaciones y contactos marítimos en el Mediterráneo occidental. Elementos de arqueología prehistórica litoral e insular », Cuadernos de Arqueología Marítima, 1, p. 115-138.

ONRUBIA-PINTADO J., 1997 - « Des marins de fortune aux Fortunées il y a trois mille ans ? Quelques considérations sur le bassin de Tarfaya (Sahara nord-atlantique) à l'aube du premier millénaire av. J.-C. et le problème de la colonisation de l'archipel canarien », Antiquités africaines, 33 (Hommages à Georges Souville, 1), p. 25-34. 
ROJO GUERRA M. A., BRAVO NIETO A., BELLVER GARRIDO J., GARRIDO PENA R., GARCIA MARTíNEZ DE LAGRÁN I. et GÁMEZ GÓMEZ S, 2006. - Una mirada al pasado... la prehistoria de las Islas Chafarinas, Melilla, Instituto de Cultura Mediterránea.

TEJERA GASPAR A. et CHÁVEZ ÁLVAREZ M. E., 2011 - « Fenicios y púnicos en las Islas Canarias. Un problema histórico y arqueológico », in J. C. Domínguez Pérez, Gadir y el Círculo del Estrecho revisados. Propuestas de la arqueología desde un enfoque social (Monografías Historia y Arte), Cadix, Servicio de Publicaciones de la Universidad de Cádiz, p. 257-269.

VERNET R., 2007 - Le Golfe d'Arguin de la préhistoire à l'histoire. Littoral et plaines intérieures (Coll. PNBA, 3), Nouakchott, Parc National du Banc d'Arguin.

INDEX

Mots-clés : Commerce, Economie, Navigation, Néolithique, Néolithisation, Préhistoire 